lation coefficient for the sun-spot effect on the diurnal-variation (range, average departure, Fourier combined amplitude) of the potential gradient for various stations is about 0.8 ; for Ebro and Eskdalemuir it exceeds 0.9 . The reason that Dr. Chree gets somewhat unsatisfactory results from certain diurnal data at Kew is partly because of the fact, already mentioned, that $\mathrm{Kew}$ is not a favourable station for the most successful study of world effects. However, applying a formula similar to (I) to the Kew series I898-I909, Dr. Chree's correlation coefficient for the sun-spot relation of his quantity $c_{r}$ (combined amplitude of the 24-hour and I2-hour waves of the Fourier series) is increased from 0.46 to $0.77 ; s=+0.50$ per cent., and $t=-2 . \mathrm{II}$ per cent. of $c_{r}$.

The sun-spot influence is also shown in the annual variation of the potential gradient at Ebro, for the period I9IO-I92 I; the correlation coefficient is $0.7 \mathrm{I}$.

General Conclusion.-The relationship between sun-spot activity and atmospheric electricity turns out to be, for locally undisturbed stations, as definite as in the case of terrestrial magnetism; the sun-spot influence on the periodic variations of the atmospheric potential-gradient is, in general, as great as on the periodic variations of terrestrial magnetism; and as concerns the effects on the absolute values, the sun-spot influence is about 300 times greater in atmospheric electricity than in terrestrial magnetism. The potential gradient of atmospheric electricity, and, presumably, the earth's total negative electric charge, is furthermore subject to an annual or secular change, which may vary in magnitude and sign from one sun-spot cycle to another.

LOUIS A. BAUER.

Department of Terrestrial Magnetism, Carnegie Institution of Washington, Washington, D.C., June 7 .

\section{Use of Yeast Extracts in Diabetes.}

In a previous letter to Nature of March Io (III, p. 327 ) we stated that we had obtained from yeast an insulin-like substance which had the effect of lowering the blood sugar of normal animals. Later we described the beneficial effect of this extract on some cases of diabetes mellitus (Brit. Med. Journ. i. p. 7II, I923). We soon found that the activity of the extract from different samples of yeast varied very widely. The results of these experiments will be published at a later date in conjunction with Dr. H. B. Hutchinson. In this connexion it is of interest to note that Collip (Proc. Soc. of Exp. Biol. and Med., 20, p. $32 \mathrm{I}, \mathrm{r923}$ ) reports numerous failures before he succeeded in obtaining an active extract from yeast, and later Funk and Corbitt (Proc. Soc. of Exp. Biol. and Med., 20, p. 422, 1923) have met with similar variability.

We have recently obtained from the action of micro-organisms other than yeast extracts which have a very considerable power of lowering the blood sugar of normal animals to a point where convulsions occur. That the convulsions were not due to a toxic effect is shown by the fact that they were relieved by injection of glucose. The extract like that from yeast caused the blood sugar to be lowered for a much longer time than when insulin was used. Whether these extracts will be of practical importance remains to be decided. Experiments are being directed to this end.

\section{B. WINTER.} W. Sмith.

Biochemical Laboratory, Cambridge, July 20 .

\section{Tenacity of Life of an Eel.}

I HAVE lately had occasion to notice a further proof of the tenacity of life exhibited by the eel, which may perhaps be of interest.

A correspondent in America, Mr. L. L. Mowbray, of the Aquarium, Battery Park, New York City, has kindly sent me from time to time specimens of elvers ${ }^{1}$ of the American eel, preserved in formol, for investigation purposes. Quite recently, a parcel from Mr. Mowbray was delivered at the laboratory here. Greatly to our surprise, however, instead of elvers preserved in formol as usual, it proved to contain a single specimen very much alive.

The little eel was enclosed in a small glass bottle (quarter-litre size), which had been corked and waxed so as to render it perfectly air-tight, and the bottle again enclosed in one of the tin cylinders commonly used in the United States for sending natural history specimens by post. The tiny creature had thus made its voyage across the Atlantic in complete darkness, and without any renewal of air in the 200 c.c. of water in which it was originally placed.

The postmarks showed that it had left New York on April 19, and arrived in Copenhagen on May 19, 1923. It has now been transferred to a small aquarium, where it is still alive and active, to all appearances in excellent form after its lengthy journey.

Evidently, then, the American fresh-water eel is by no means inferior to its European cousin in respect of endurance and tenacity of life.

I may add that we have, at the Laboratory here, two live adult specimens of the American eel. They have been in our aquaria since I9I4, when we brought them home, as elvers, from Santa Cruz, in the West Indies. They, however, made the journey in an open beer bottle, with frequent changes of water, and were thus not subjected to so severe a test of endurance as the specimen above mentioned.

Carlsberg Laboratory, Copenhagen,

Johs. SCHMIDT. June 28 .

\section{Adsorption on Soil-Grains.}

THE recently published work by Messrs. J. Hendrick and G. Newlands (Journ. Agric. Sci., January 1923) on the mineral particles in the coarser grades of the "fine earth" separated from soils was noticed in NATURE of June $9, p .736$, and it was remarked that "the study of adsorptive reactions should not be entirely restricted to the colloidal field."

It is of interest to note that the United States Department of Agriculture took up this question last year $_{3}$ and its Bulletin No. $\mathrm{II}_{22}$ (October 2I, I922) records the work of Messrs. M. S. Anderson, W. H. Fry, P. L. Gile, H. E. Middleton, and W. O. Robinson, on "Absorption by colloidal and non-colloidal soil constituents." The authors worked on material finer than $2 \mathrm{~mm}$. in diameter, which, in common with so many experimenters, they call "the soil," by an unfortunate restriction of the term. This earth is separated, preferably by centrifugal methods, into three grades, $2 \cdot 000-0.050 \mathrm{~mm}$., $0.050-0.001 \mathrm{~mm}$., and less than $0^{\circ}$ oor $\mathrm{mm}$., the last being styled colloidal. In testing the relative powers of adsorption on (or absorption by) these grades, it was justly felt that samples really free from colloidal matter could be best obtained by crushing unaltered minerals. In

1 The youngest stages of eel-fry which make their way up into fresh water are called elvers. 